\title{
MAGNETIC STRUCTURE CALCULATION ON A FERRIMAGNETIC FRUSTRATED COMPOUND
}

\author{
J. Linares $\left({ }^{2}\right)$, J. M. Greneche $\left({ }^{1}\right)$ and F. Varret $\left({ }^{1}\right)$ \\ $\left({ }^{1}\right)$ Laboratoire de Spectrométrie Mössbauer (UA 807), Université du Maine F72017 Le Mans, France \\ $\left({ }^{2}\right)$ Pontifica Univerdisad Catolica, Lima, Peru
}

\begin{abstract}
The magnetic structure of crystalline materials can be calculated by a procedure which consists in minimizing the magnetic interaction energy by a gradient method. Calculations were carried out on the weberite structural system, magnetically frustrated. The present results agree the experimental data of a ferrimagnetic structure, previously evidenced by neutron diffraction technique.
\end{abstract}

\section{Introduction}

During the last decennies, many papers were devoted to the determination of magnetic structures of crystalline compounds either from experimental data or from simulations. The neutron diffraction technique has proved to be a valuable tool by analysing the differences between spectra recorded above and below the magnetic ordering temperature. The determination of the magnetic structure (magnitudes and directions of the magnetic moments) can take advantage of symmetry analysis, according to Bertaut's method [1]. Also, the thermal dependance can be predicted using the molecular field approximation, assuming exchange interactions with unlimited range and supposing the magnetic ions to be on a Bravais lattice [2]. More recently, simulations of magnetic structures in crystalline compounds were obtained by a program based upon the Metropolis method, using exchange interactions values and symmetry space group [3]. Both methods allow for non-collinear structures in frustrated systems.

In the present paper, we show how the simulation of such frustrated magnetic structures can be obtained by a gradient method, needing rather short computer times. Since temperature does not appear explicitely, the present results specifically apply to the low temperature magnetic properties.

\section{The methodology}

The simulation of the magnetic structure consists in rotating the magnetic moments using the gradient method till the total magnetic interaction energy is minimum.

The calculations were carried out on volumes including the elementary cell and the magnetic nearest neighbours displayed around the origin cell. The initial magnetic configuration is obtained by randomly generating a set of magnetic moment directions con- sistent with the magnetic space group (already known or proposed according to [1]).

The magnetic interaction energy of the elementary cell containing $N$ spins is given by the following Hamiltonian

$$
\begin{aligned}
H=-\frac{1}{2} \sum_{i, j}^{N} & J_{i j} \mathbf{S}_{i} \mathbf{S}_{j}-\sum_{i} D_{i}\left(S_{z}\right)^{2}+ \\
& +\sum_{i, j}^{N}\left\{\frac{\mathbf{S}_{i} \mathbf{S}_{j}}{r_{i j}^{3}}-3 \frac{\mathbf{S}_{i} \mathbf{r}_{i j} \mathbf{S}_{j} \mathbf{r}_{i j}}{r_{i j}^{5}}\right\}-\sum_{i=1}^{N} \mathbf{H} \mathbf{S}_{i}
\end{aligned}
$$

were $J_{i j}(<0)$ is the antiferromagnetic exchange integral between $S_{i}$ and $S_{j}$, the pairs of considered neighbours spins, $D_{i}$ represents the single-ion anisotropy term, the anisotropic dipole-dipole interaction being the following term (where $2 r_{i j}$ is the distance between $S_{i}$ and $S_{j}$ ) and $H$ an applied field, if any.

Heisenberg type moments were considered in the present study. Spin reorientations were performed by an iterative procedure: at each step, each spin is examined individually and rotated according to the torque due to its magnetic neighbours. The equilibrium state corresponding to the final magnetic configuration is yielded when the magnetic energy of all the system is close to be constant. In absence of anisotropic terms or dipole interactions, one spin has to be fixed, and the magnetic structure is obtained save for a rotation.

After optimizing the calculation procedure, mainly avoiding that the magnetic system is in a secondary minimum, the magnetic ground state of the system at $0 \mathrm{~K}$ was obtained with short computer times (typically $\sim 500$ successive iterations per spin compared to $\sim 5000$ Monte Carlo cycles per spin at each temperature in [3]).

So, the first aim of the present simulation procedure, i.e. the calculation of the magnetic configuration of a crystalline system, knowing the magnetic space group, the exchange values $J_{i j}$ and the magnitudes of the magnetic moments, was achieved. At the reverse, 
this method can be conveniently applied to determine the values of $J_{i j}$ from the experimental magnetic structure.

On can note that in the direct procedure, when the magnetic space group is unknown, a larger number of magnetic moments has to be taken into account: the final magnetic configuration is unaffected, but the computer time is largely increased.

\section{Results and discussion}

This simulation method was applied to $\mathrm{AFeF}_{5}\left(\mathrm{H}_{2} \mathrm{O}\right)_{2}$ crystalline compounds belonging to the inverse weberite group; their crystallographic structure (S.G. Pmma) exhibits cationic triangles leading to frustrated antiferromagnetic interactions, as shown in figure la. Neutron diffraction experiments in the case of $\mathrm{MnFeF}_{5}\left(\mathrm{H}_{2} \mathrm{O}\right)_{2}$ and by High Field Mössbauer Spectroscopy (HFMS) have been recently reported ([4, 5] respectively).

Calculations were performed in the case of $\mathrm{MnFeF}_{5}\left(\mathrm{H}_{2} \mathrm{O}\right)_{2}$ - containing non-anisotropic ions - by considering interactions between first-nearest neighbours. The final configuration shows an iron sublattice strongly canted, in contrast with the manganese sublattice weakly canted; the angles between $\mathrm{Fe}^{3+}-\mathrm{Fe}^{3+}$ and $\mathrm{Fe}^{3+}-\mathrm{Mn}^{2+}$ moments compare well to the experimental data obtained from neutron diffraction technique (the spin arrangement is shown in Fig. 1b). At the reverse, to fit the experimental data given in [4], the exchange integral values $J_{\mathrm{Mn}-\mathrm{Mn}}, J_{\mathrm{Fe}-\mathrm{Fe}}$ and $J_{\mathrm{Fe}-\mathrm{Mn}}$ were estimated at $-0.9 \mathrm{~K},-11.6 \mathrm{~K}$ and $-4.6 \mathrm{k}$ respectively by the present procedure. These values are close to magnetic susceptibility data $\left(J_{\mathrm{Fe}-\mathrm{Fe}}\right.$ $\left(\mathrm{ZnFeF}_{5}\left(\mathrm{H}_{2} \mathrm{O}\right)_{2}\right)=-11.8 \mathrm{~K}$ [4]) and Monte Carlo calculations $\left(J_{\mathrm{Mn}-\mathrm{Fe}} / J_{\mathrm{Fe}-\mathrm{Fe}}\left(\mathrm{MnFeF}_{5}\left(\mathrm{H}_{2} \mathrm{O}\right)_{2}\right)=0.49\right.$ [3]).

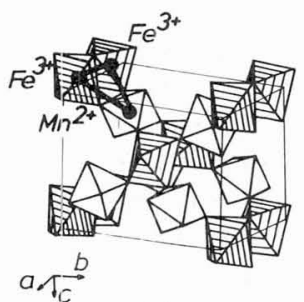

(a)

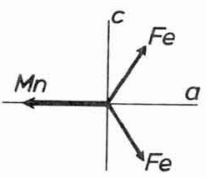

(D)
Fig. 1. - (a) Crystallographic view of $\mathrm{MnFeF}_{5}\left(\mathrm{H}_{2} \mathrm{O}\right)_{2}$ : a frustrated cationic triangle is outlined. (b) Spin arrangement projected in the $a, c$ plane.
Due to its major advantage of short computer times, this method is currently applied to the case of external magnetic field (to interpret the progressive rotation of the iron moments on $\mathrm{MnFeF}_{5}\left(\mathrm{H}_{2} \mathrm{O}\right)_{2}$ evidenced by H.F.M.S. [5]) and to the case of amorphous systems which requires a high number of magnetic moments. In the latter case, preliminary calculations on handbuilt and simulated random corner-sharing octahedral networks $\mathrm{AB}_{3}$ [6] with an highly frustrated topology, indicate the absence of short range magnetic order [7] consistent with the speromagnetic behaviour of $\mathrm{FeF}_{3}$, evidenced by HFMS [8].

Additional results concerning crystalline and amorphous systems exhibiting non-collinear magnetic structures will be detailed in a following paper.

[1] Bertaut , E. F., Magnetism III, Eds. G. T. Rado and H. Suhl (Academic Press, New York) 1963, p. 149.

[2] Villain, J., J. Phys. Chem. Solids 11 (1959) 303.

[3] Lacorre, P. and Pannetier, J., J. Magn. Magn. Mater. 71 (1987) 63.

[4] Laligant, Y., Calage, Y., Torres-Tapia, E., Greneche, J. M., Varret, F. and Ferey, G., J. Magn. Magn. Mater. 61 (1986) 283;

Laligant Y., Pannetier, J., Leblanc, M., Labbe, P., Heger, G. and Ferey, G., Z. Kristallogr. 181 (1987) 1 ;

Laligant, Y. and Ferey, G., J. Solid State Chem., to appear.

[5] Greneche, J. M., Linares, J., Varret, F., Laligant, Y. and Ferey, G., J. Magn. Magn. Mater. 73 (1988) 115.

[6] Greneche, J. M., Teillet, J. and Coey, J. M. D., $J$. Non Cryst. Solids 83 (1986) 27; J. Phys. France 48 (1987) 1709;

Coey J. M. D. and Murphy P. J. K., J. Non Cryst. Solids 50 (1982) 125.

[7] Greneche, J. M. and Coey, J. M. D., unpublished results.

[8] Ferey, G., Varret, F. and Coey, J. M. D., J.Phys. $C 12$ (1979) L531;

Greneche, J. M., Varret, F., Leblanc, M. and Ferey, G. Solid State Commun. 63 (1987) 435. 